\title{
Intergrading effect of investigation of Carbon monoxide adsorbing on Al-doped SiC(001) Surface
}

\author{
Shaohua Zhang, Anhong Hou, Zhengxin Yan, Meiling Men \\ College of Science, Xi'an university of science and technology, 710000 \\ Xi'an, P.R. China \\ e-mail: zshxust@163.com, houanhong1@126.com, zhengxinyan163@163.com, \\ mmlxust@163.com
}

Keywords: deep groove ball bearing; quasi-dynamics; permissible wear; wear-life

\begin{abstract}
We study the adsorption integrated effects of CO with different coverage $(1 / 20,1 / 10$, $3 / 20,1 / 5)$ on doped-Al-SiC(001) surface by using the generalized gradient approximation (GGA) based on density functional theory (DFT). The PDOS data reveal that p-electrons of $\mathrm{Al}$ increase the interfacial activities though $\mathrm{Al}$ atoms change the geometrical characteristic of SiC slightly. The changing geometric structure of the adsorbed $\mathrm{CO}$ exhibits the integrated effects of the increasing numbers of the adsorbed molecules. The electron density difference shown that the repulsion of the neighbor the same atoms the next-neighbor effect affects the electronic distribution of $\mathrm{C}$ and $\mathrm{O}$ atoms. The neighbor and next-neighbor effect of the adsorbed $\mathrm{CO}$ will change the role of the $\mathrm{C}$ atom of CO molecule of acceptor or contributor causing for the change of O-C-Al.
\end{abstract}

\section{INTRODUCTION}

Owing to the wide band gap, high breakdown filed, and high heat conductivity, SiC (silicon carbide) has outstanding device potential for high power, high temperature and high frequency application.[1-4] The related chemical and physical properties of SiC are fundamental importance of researchers who devote their efforts to innovative applications. Motivated by chemical and physical important processes such as chemical vapor deposition, doped film growth, interface interaction, formation of stable low dielectric, and accompanied by a more general interest in the effect of absorbed gas molecules that is applied to research and develop the gas detector for CO, NH3 and methane in ultra-low concentration and harsh environment,[5] the interaction of inner adsorbed molecules has been studied by using density functional theory (DFT) quite extensively in past.[6-11]

Doping elements is a common method to improve the adsorption performance of semiconductors.[12] The properties of SiC doping have been extensively studied in theory computation. $\mathrm{Li}$ [13] has investigated the electronic structure in Al-doped 4H-SiC. They found the Fermi level into the valence band and the energy gap broadening slightly after aluminum atoms doped, which implies that doping can indeed adjust the properties of semiconductor. Dou et al. [14] have studied the electronic properties of SiC doped with different number of $\mathrm{Ni}$. The result shows that a certain number of new impurity energy level is introduced in the energy band gap owning to $3 \mathrm{~d}$ orbits of $\mathrm{Ni}$, though the analysis of the DOS, electron density difference and Mulliken overlap populations conformed that the $\mathrm{Ni}$ atoms are interacting with their neighboring host $\mathrm{C}$ atoms. The adsorption of gases on the surface of gas sensors is a complex process, there are many factors affecting the adsorption effect. Fortunately, many researchers have carried on the related investigation in this filed. Cheng [15] has researched the CO molecule adsorption on SiC surface. Concluded that $\mathrm{CO}$ molecule make $\mathrm{SiC}$ exhibits metal characters when it is adsorbed on the surface, this make a clue for the design of gases sensitive device. Zahra [16] suggested that CO interaction amongst themselves (intermolecular) when CO adsorbed on the surface at coverage of $0.5 \mathrm{ML}$, which arises the interest in the study of the intergrading effects on adsorption properties such as the space geometry, band gap and adsorption energy etc. However, there still lacks of detailed 
knowledge of considering the intergrating effect of adsorbed molecules when interacting with aluminum doped interface.

In this work, we will focus our attention on two related effects, doped-Al and the intergrating effect of CO adsorbed. Firstly, in order to understand the mechanism and effect of the atoms doped,we chose nonmagnetic element aluminum doped on $4 \mathrm{H}-\mathrm{SiC}(001)$ surface to establish the doping model since doped aluminum can form a p-type doped semiconductor which can improve the conductive capacity. Second, polar molecules, CO, with different coverage were chosen to investigate the neighbor effect and the next neighbor effect of absorbed molecule.

\section{Calculation models and methods}

In this paper, the intergrating effect of $\mathrm{CO}$ molecule on doped Al-SiC was performed with the program package CASTEP in Materials Studio. As been well known, valence electron configureurations of $\mathrm{Si}, \mathrm{C}, \mathrm{O}$ and $\mathrm{Al}$ were selected as $3 \mathrm{~s}^{2} 3 \mathrm{p}^{2}, 2 \mathrm{~s}^{2} 2 \mathrm{p}^{2}, 2 \mathrm{~s}^{2} 2 \mathrm{p}^{4}$ and $3 \mathrm{~s}^{2} 3 \mathrm{p}^{1}$ respectively. For a better adsorption geometrical structure characters, the generalized gradient approximation (GGA) with the PW91 function for the exchange correlation energy calculation was chosen in this work. [17-19] With the testing experiences, the supercell was set to $5 \times 4 \times 1$ and the vacuum thickness was set to $12 \mathrm{~A}$ along the Z-aixs. For satisfying the requirement of computation, the accuracy of convergence was set to $1 \times 10-5 \mathrm{eV} /$ atom, the stress accuracy of convergence in crystal was set to $0.02 \mathrm{GPa}$ and the maximum displacement was set to $0.002 \mathrm{~A}$ with the cut-off energy of plane wave was set to $400 \mathrm{eV}$ in iterative program in this calculation. The optimization step would have stopped until all the convergence conditions were satisfied.

Figure. 1 shows the supercell structure of $(5 \times 4 \times 1) 4 \mathrm{H}-\mathrm{SiC}(001)$ and $\mathrm{Al}$-doped $4 \mathrm{H}-\mathrm{SiC}(001)$ structure before the optimization. In Figure. 1, the yellow color ball represents silicon atoms, the gray balls are carbon atoms and the pink ones are aluminum atoms respectively. On $(5 \times 4 \times 1)$ Al-doped $4 \mathrm{H}-\mathrm{SiC}(001)$ surface, the doping method was that making aluminum atoms replace the silicon atoms on $\mathrm{SiC}(001)$ crystal surface forming a substitution type doping. Doping ratio of aluminum and whole atoms on the surface is 1:4.

As shown in Figure 2, we designed the adsorption molecule, CO, with a perpendicular to the interface for a convenient observation on the changing absorbing angles with the intergrating effect.

In Figure. 2, the length of $\mathrm{C}-\mathrm{O}$ band was set to $1.13 \AA$ and $\mathrm{C}-\mathrm{Al}$ bond was $1.92 \AA$ which was similar with those parameters. For the sake of discussion, we use the red numbers to mark the CO molecules.[20-22]
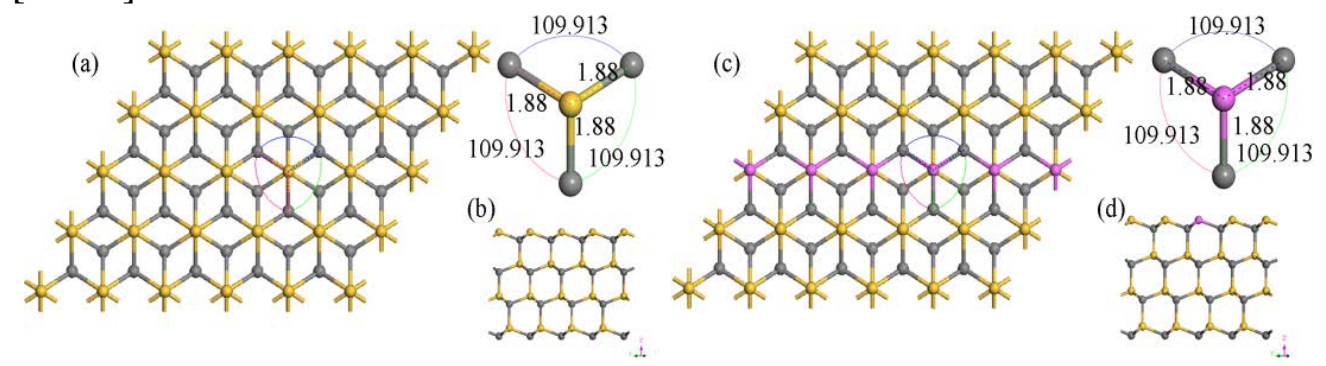

Figure 1 . The $(5 \times 4 \times 1) \mathrm{SiC}(001)$ structure and Al-doped $4 \mathrm{H}-\mathrm{SiC}(001)$ structure: (a) top view of $4 \mathrm{H}-\mathrm{SiC}(001)$ structure, (b) perspective view of $4 \mathrm{H}-\mathrm{SiC}(001)$ structure, (c) top view of Al-doped $4 \mathrm{H}-\mathrm{SiC}(001)$ structure and $(\mathrm{d})$ perspective view of Al-doped $4 \mathrm{H}-\mathrm{SiC}(001)$ structure. 


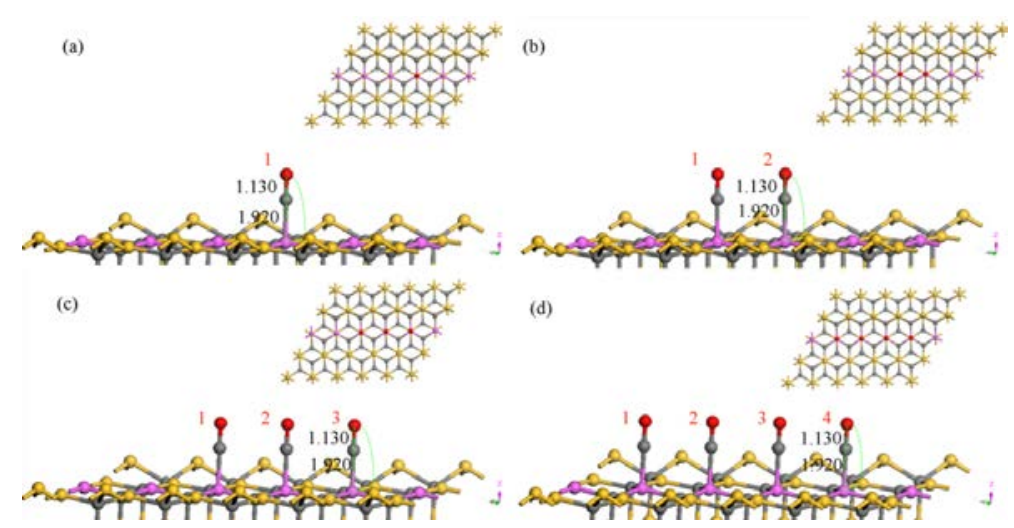

Figure 2. CO adsorbed on different surface at different coverage before optimization: (a) $1 \mathrm{CO}$ on $\mathrm{Al}$-doped 4H-SiC(001) surface, (b) $2 \mathrm{CO}$ on Al-doped 4H-SiC(001) surface, (c) $3 \mathrm{CO}$ on Al-doped $4 \mathrm{H}-\mathrm{SiC}(001)$ surface, (d) $4 \mathrm{CO}$ on $\mathrm{Al}$-doped $4 \mathrm{H}-\mathrm{SiC}(001)$ surface.

\section{Results and discussion}

\subsection{Structual changes of $\mathrm{SiC}(001)$ structure and Al-doped SiC(001) structure.}

For convenience of comparison, the structure parameters of $4 \mathrm{H}-\mathrm{SiC}(001)$ and Al-doped $4 \mathrm{H}-\mathrm{SiC}(001)$ optimizated are shown in Figure. 3. As shown in Figureure, the length of $\mathrm{C}-\mathrm{Al}$ bond of Al-doped $4 \mathrm{H}-\mathrm{SiC}(001)$ structure is longer than the C-Si bond of $4 \mathrm{H}-\mathrm{SiC}(001)$ structure which means that the weaken bond will drop a hint on the more active interface. The volume of structure changed from $3301.08 \AA^{3}$ to $3312.236 \AA^{3}$ obtained by calculation, due to the mutual repulsion between the aluminum atoms and the neighboring carbon atoms and the radius of the aluminum

atom $(1.8 \AA$ ) which is larger than the radius of silicon atom $(1.1 \AA)$. The total energy of $(5 \times 4 \times 1)$ $\mathrm{SiC}(001)$ structure has decreased from $20977.5 \mathrm{eV}$ to $20728.02 \mathrm{eV}$ after doping aluminum atoms. We notice that the lattice parameters of Al-doped $\mathrm{SiC}(001)$ optimized were very similar to the structure of $\mathrm{SiC}(001)$, which means that the aluminum atoms doped has slightly influence on the structure of $\operatorname{SiC}(001)$.

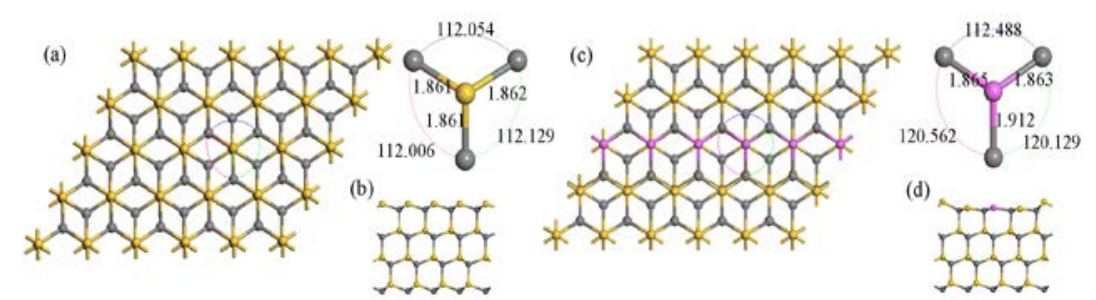

Figure 3. The optimized $(5 \times 4 \times 1) \mathrm{SiC}(001)$ structure and Al-doped 4H-SiC(001) structure: (a) top view of optimization $4 \mathrm{H}-\mathrm{SiC}(001)$ structure, (b) perspective view of optimization $4 \mathrm{H}-\mathrm{SiC}(001)$ structure, (c) top view of optimization Al-doped 4H-SiC(001) structure and (d) perspective view of optimization Al-doped 4H-SiC(001) structure.

In order to study the electronic structure properties of the aluminum atoms doped clearly, we calculated the partial density of states (PDOS) of $(5 \times 4 \times 1) \mathrm{SiC}(001)$ and $(5 \times 4 \times 1)$ Al-doped SiC $(001)$ surface which was illustrated in Figure. 4. As can be seen in Figure. 4(a), the valence band (VB) of $(5 \times 4 \times 1) \mathrm{SiC}(001)$ surface can be divided into two groups: the low energy VB has a width of $5.3 \mathrm{eV}$, ranging from $-16.6 \mathrm{eV}$ to $-11.3 \mathrm{eV}$, mainly originating from $\mathrm{C} 2 \mathrm{~s}$ orbital electrons, while high energy VB locates between $-9.7 \mathrm{eV}$ to $0 \mathrm{eV}$ primarily from $\mathrm{C} 2 \mathrm{p}$ orbital electrons. The conduction band (CB) ranging from $1.6 \mathrm{eV}$ to $3.5 \mathrm{eV}$ has a width $1.9 \mathrm{eV}$ mainly contributes from Si 3p. Compared Figure. 4(a) with Figure. 4(b), we find when three aluminum atoms substitute for three silicon atoms, the $\mathrm{CB}$ appeals a new peak ranging from $0.8 \mathrm{eV}$ to $2.7 \mathrm{eV}$, which mainly originates from the $\mathrm{Al} 3 \mathrm{p}$ orbits. Thus, the band gap $\left(E_{g}\right)$ becomes much narrower, which means that after the aluminum atoms were doped on SiC surface, the surface becomes more active substance for an 
adsorption system.
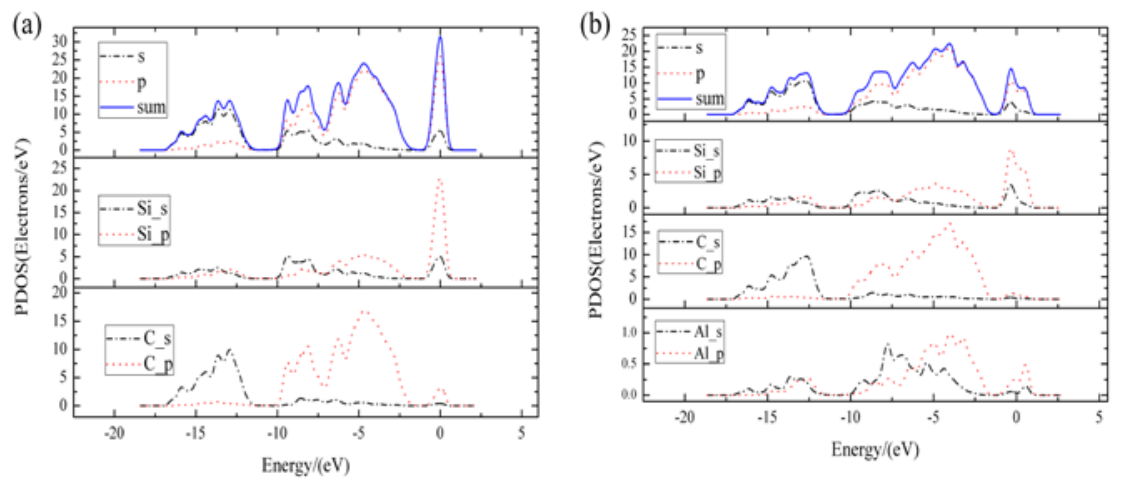

Figure 4. The density of states of supercell: (a) $(5 \times 4 \times 1) \mathrm{SiC}(001)$; (b) $(5 \times 4 \times 1)$ Al-doped $\mathrm{SiC}(001)$.

To further understand how the charge transfers of atoms and the bond situation between atoms, we calculated the electrons difference density of $\mathrm{SiC}(001)$ and Al-doped $\mathrm{SiC}(001)$ surfaces. The electrons difference density of $(5 \times 4 \times 1)) \mathrm{SiC}(001)$ and $(5 \times 4 \times 1)$ Al-doped $\mathrm{SiC}(001)$ surface are showed in Figure. 5. The covalent characteristic can be obviously seen in Figure. 5(a). The nucleus is bound by the density charge shared between silicon and carbon atom. On the Al-doped SiC(001) surface, as is showed in the Figure. 5(b), the electron shifts toward aluminum atoms to compensate for the hole introduced by aluminum doping. The double peaks over Fermi face is from the p orbital of $\mathrm{Si}$ and $\mathrm{Al}$, which is mainly from their $\pi^{*}$ orbits, as shown in Figure. 4(b). The PDOS data from the Al-doped $\mathrm{SiC}$ reveals that the doped aluminum atoms cause for the double peaks of Al-doped $\mathrm{SiC}$, which is a clearly characteristic of delocalized pi-electrons. The electronics density difference of $\mathrm{SiC}$ exhibits that $\mathrm{Si}-\mathrm{C}$ has bonded while Al-C also bonded although it was weaken. An interesting phenomenon is that the doped $\mathrm{Al}$ polarized the next neighbor silicon, as shown in Figure. 5(b).

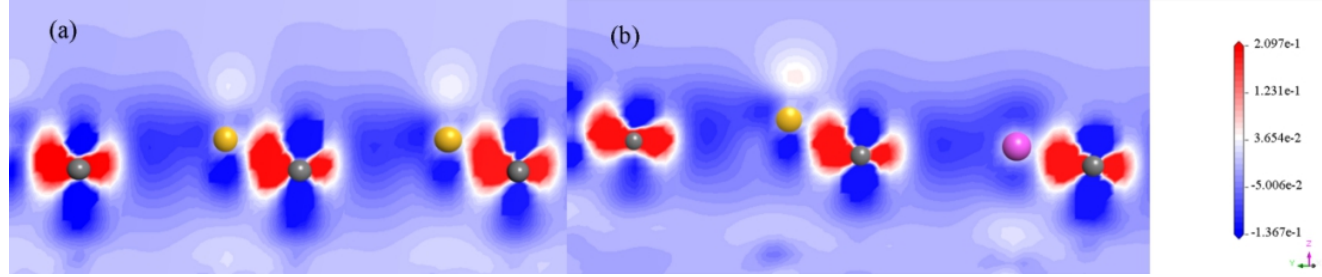

Figure 5. Figureure of electrons difference of each surface: (a) Electrons density difference of $\mathrm{SiC}(001)$, (b) Electrons density difference of Al-doped SiC(001).

\subsection{Adsorption characteristic of different number CO molecules on Al-doped 4H-SiC(001) surface.}

To clearly display the spatial change of the adsorbed molecules, the geometric structure for different coverage of $\mathrm{CO}$ absorbed on Al-doped $\mathrm{SiC}(001)$ surface has been constructed and the bond structure change was showed in Figure. 6. The C-O bond length was $1.208 \mathrm{~A}$, C-Al bond was $2.188 \mathrm{~A}^{\circ}$ and angle change of O-C-Al decreased $39.924^{\circ}$ and $\mathrm{C}-\mathrm{Al}-\mathrm{Si}$ decreased $43.812^{\circ}$ at the coverage of $1 / 20$. The bond length of $\mathrm{C}-\mathrm{O}$ was $1.174 \AA$ to $1.175 \AA$, C-Al bond was $2.036 \AA$ to $2.038 \AA$, the angle change of $\mathrm{O}-\mathrm{C}-\mathrm{Al}$ was decreased $21.613^{\circ}$ and $23.189^{\circ}$ and the angle change of C-Al-Si was decreased $19.014^{\circ}$ and $20.995^{\circ}$ at coverage of $1 / 10$. The bond length of C-O was $1.163 \AA$ to $1.177 \AA$, C-Al bond was $2.005 \AA$ to $2.010 \AA$, the angle change of O-C-Al is decreased $14.708^{\circ}, 10.922^{\circ}$ and $22.864^{\circ}$ and the angle change of $\mathrm{C}-\mathrm{Al}-\mathrm{Si}$ is $5.646^{\circ}, 4.997^{\circ}$ and $6.681^{\circ}$ at coverage of $3 / 20$. The bond length of $\mathrm{C}-\mathrm{O}$ was $1.168 \AA$ to $1.176 \AA$, C-Al bond was $2.041 \AA$ to $2.071 \AA$, the 
angle change of $\mathrm{O}-\mathrm{C}-\mathrm{Al}$ is decreased $22.894^{\circ}, 11.181^{\circ}, 21.782^{\circ}$ and $20.447^{\circ}$ and the angle change of $\mathrm{C}-\mathrm{Al}-\mathrm{Si}$ is $22.99^{\circ}, 18.558^{\circ}, 20.186$ and $19.003^{\circ}$ at coverage of $1 / 5$. In the process of analysis the various of geometry structure, amazingly, we have found some interesting adsorption pattern. In summary, those special parameters of $\mathrm{C}-\mathrm{O}, \mathrm{C}-\mathrm{Al}$ and $\mathrm{O}-\mathrm{C}-\mathrm{Al}$ will decrease with the increasing adsorbed $\mathrm{CO}$. We also find an odd-even selective adsorption growing model, i.e. the spatial change with even $\mathrm{CO}$ molecules exhibits the same characterization, and geometry will shows different rules with odd molecules adsorbed on the interface. The intrinsic intergrading effect of related electrons will be discussed from the view of electron density difference in the following section.

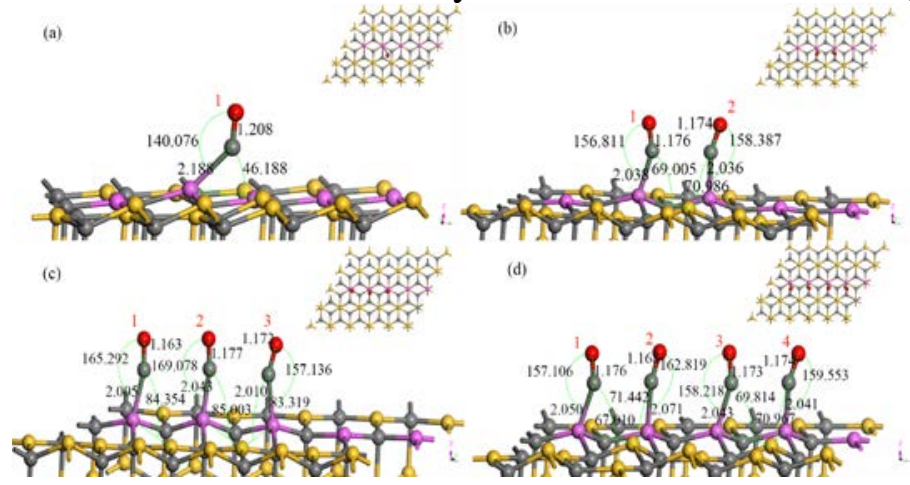

Figure 6. The geometric structure of $\mathrm{CO}$ molecules adsorbed on $\mathrm{Al}$-doped $\mathrm{SiC}(001)$ surface at different coverages (a) coverage of 1/20 ML, (b) coverage of 1/10 ML, (c) coverage of 3/20 ML and (d) coverage of $1 / 5 \mathrm{ML}$.

We would like to calculate the adsorption energy $\left(E_{a d}\right)$ to verify our conjecture on the role among CO molecules. The adsorption energy of CO on aluminum doped SiC(001) surface can be deduced by Eq (1). [23]

$E_{a d}=-\frac{1}{N}\left(E_{\text {SiC.CO }}-E_{\text {SiC }}-N^{*} E_{C O}\right)$

Here, $\mathrm{N}$ represents the number of $\mathrm{CO}$ molecules adsorbed on $\mathrm{SiC}$ or Al-doped $\mathrm{SiC}$ surface, The $E_{a d}$ is the adsorption energy of $\mathrm{CO}$ molecules on $\mathrm{SiC}(001)$ surface. If the value of $E_{a d}$ is positive, it means the adsorption is an exothermic reaction, and the bigger the value is, the stronger the reaction is.

With Eq (1), the adsorption energy at different coverage of CO was calculated and displayed in Figure. 7. The adsorption energy of CO on Al-doped SiC(001) surface at coverage of 1/20 ML, 1/10 ML, 3/20 ML and 1/5 are $2.0861 \mathrm{eV}, 1.7012 \mathrm{eV}, 1.5894 \mathrm{eV}$ and $1.6902 \mathrm{eV}$, respectively. The calculated $E_{a d}$ versus the CO coverage for the adsorption of $\mathrm{CO}$ on Al-doped SiC(001) surface indicated that the intergrading effect of the adsorbed CO is weakening the interaction of $\mathrm{CO}$ with the interface. We found also that there are the optimized coverage for the molecules adsorption system but there are the same adsorption energy for two even molecular adsorption system(i.e.1/10 and 1/5 ML), which was consistent with the structure analysis above.

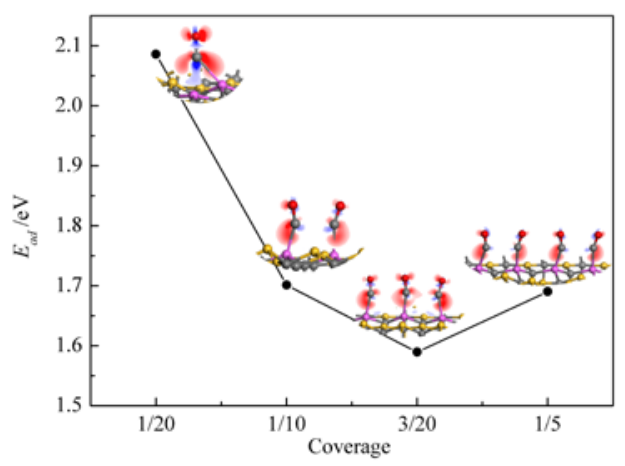

Figure 7. The calculated $E_{a d}$ versus the CO coverage for the adsorption of CO on Al-doped $\mathrm{SiC}(001)$ surface. 
PDOS of CO molecules and Al-doped $\mathrm{SiC}(001)$ surface with different coverage have been calculated. The results were shown in Figure. 8 and Figure. 9. We can see that the electron peaks of $\mathrm{CO}$ molecules at free state are mainly concentrated in $-20 \mathrm{eV},-5 \mathrm{eV}, 0 \mathrm{eV}$ and $11 \mathrm{eV}$ which are mainly from the p orbital electrons, as shown in Figure. 8(a). The PDOS of CO adsorbed on Al-doped SiC(001) surface is shown in Figure. 8 (b) - (e) corresponding to the coverage at 1/20, $1 / 10,3 / 20$ and 1/5 ML, respectively. We find a tend that the electron orbital of CO molecules move to low energy level with the increasing coverage. When the coverage is $1 / 20 \mathrm{ML}$, all electron peaks of $\mathrm{CO}$ molecule orbital moved $2 \mathrm{eV}$ to low energy levels, the electron peaks of bonding orbital became wider, and peak values reduced. When coverage is 1/10 ML, the extent of movement to lower energy level is the same as coverage is $1 / 20 \mathrm{ML}$, but the difference is that the bonding orbital is wider than $1 / 20 \mathrm{ML}$ and two electron peaks appeared at $-11 \mathrm{eV}$ to $-9 \mathrm{eV}$, which means the adsorption is more stable. When coverage is $3 / 20 \mathrm{ML}$, the situation becomes different to both of the first two, all electrons orbital shifts $4 \mathrm{eV}$ to lower energy level, and the peaks value becomes higher, due to the neighbor effect among CO molecules. When coverage is $1 / 5$, we find that it's outline of PDOS almost the same as the PDOS of two CO molecule on Al-doped SiC(001) surface, which evidences the selective adsorbing growing model again. From Figure. 9, it can be concluded that PDOS of Al-doped SiC(001) surface changed little with CO molecules adsorbed, the results show that all electron peaks shift to the direction of low energy slightly and some electron peaks appear at -25.5 eV to $-22.5 \mathrm{eV}$. In addition, all the PDOS of CO and Al-doped SiC(001) surface at different coverage shows the $\mathrm{CO}$ molecules adsorbed on $\mathrm{Al}$-doped $\mathrm{SiC}(001)$ is a chemical adsorption and the neighbor effect among $\mathrm{CO}$ molecules exists in the adsorption process.

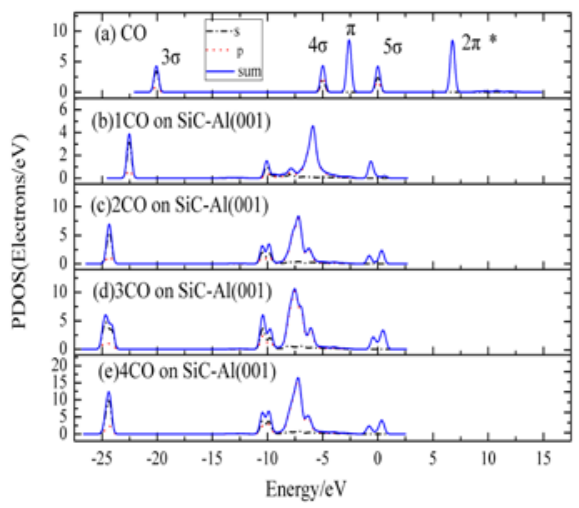

Figure 8. PDOS of CO molecules at different coverage.

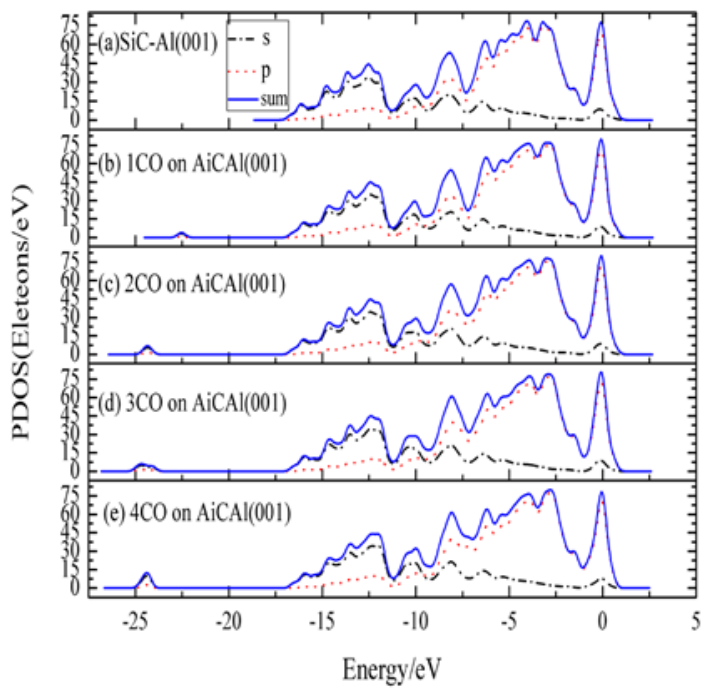

Figure 9. PDOS of Al-doped SiC(001) surface at different coverage. 
The electron redistribution and Mulliken change of related atoms for the adsorption systems are displayed in Figure. 10. When one $\mathrm{CO}$ molecule interacted with the interface, its electron density difference exhibited a pretty spatial system a bit like honeybee in Figure. 10(a), which shows special symmetric electronic distribution. The electronic repellant of the same neighbor molecules was clearly seen, at least the increasing distance between $\mathrm{O}$ atoms and their electronic cloud is so. From Figure. 10(c), we found some slight torsion occurred to electron clouds among CO molecules with the increasing adsorbing $\mathrm{CO}$ molecules, we believe it is for the combination intergrading effects of neighbor and next-neighbor molecules. From Figure. 10(d), although it exhibits a similar electronic distribution as that in Figure. 10(b), the electronic distribution of odd and even CO molecule exhibits respective similarity, which explains mainly the selective odd-even growing model. From the view of electron transferring, $\mathrm{O}$ receive $-0.41 \mathrm{eV}$ and $\mathrm{C}$ atom and $\mathrm{Al}$ atom contribute $-0.07 \mathrm{eV}$ and $1.32 \mathrm{eV}$ in Figure. 10 (a). The neighbor effect takes significant role in electron transfer when the second CO molecule was designed with a distance about 0.19 , as shown in Figure. 10(b). The significant change is that the intergrading effect transfers the role of the two C atom from contribution to receiver, 0.09 and $0.10 \mathrm{eV}$, respectively, which reveals the cause for $\mathrm{O}-\mathrm{C}$-Al decrease in this geometry. The $\mathrm{C}$ atoms also become contributor when the third CO molecule was absorbed on the interface. The electron density distribution became more harmony than that in Figure. 10(c), the acceptation and contribution of $\mathrm{C}$ and $\mathrm{O}$ atoms evidence the analysis above. Interestingly, if we take the four CO adsorbing molecules in Figure.10(d) as two groups, we can see that the neighbor effect phenomenon is more obvious than that in Figure. 10(c). In summary, from these results, we think that the chosen spacial geometries and the decreasing adsorption energy of the adsorption system determined by it's the following features, (1) the repellion of the neighbor the same atoms is the main factor for its structures and energy, (2) the other factor is from the thickening interaction of $\mathrm{C}-\mathrm{Si}, \mathrm{Si}$ from the interface, (3) the next-neighbor effect affects the electronic distribution of $\mathrm{C}$. with the increasing numbers of $\mathrm{CO}$ molecules, the intergrading effect of the neighbor and the next neighbor will been exhibited by the electron state density of $\mathrm{C}$ and $\mathrm{O}$ atoms, and indirectly displayed by the adsorption energy as discussed above.

The electron redistribution that occurs on adsorption of CO is analyzed through projected density of state diagrams, changes in populations of molecular orbitals and atomic orbitals of the CO adsorbate, and changes in electron populations of atomic orbitals of surface atoms.

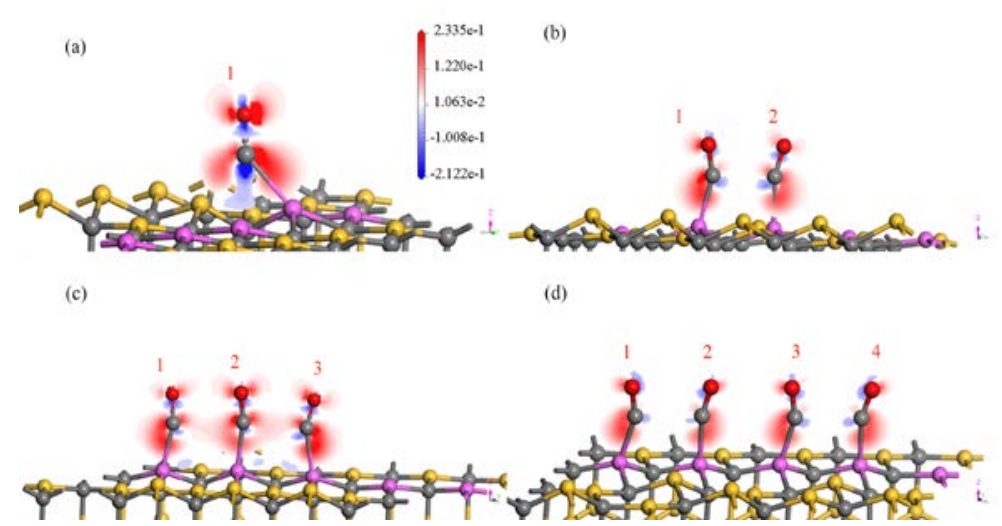

Figure 10. Figureure of electron density difference of CO molecules adsorbed on Al-doped SiC(001) surface at different coverage: (a) coverage of 1/20 ML, (b) coverage of 1/10 ML, (c) coverage of $3 / 20 \mathrm{ML}$ and (d) coverage of 1/5 ML.

\section{Conclusion}

In this presented study, doped- and no-doped $\mathrm{Al} \mathrm{SiC}(001)$ interface systems have been investigated and compared by using the first principles DFT-GGA method. It was observed that doped-Al will change the geometry structure of SiC slightly except it will significantly increase the activity of interface, which is the p-electrons of Aluminum atoms increasing the electric content 
over the Fermi level cause for the increasing activity. The PDOS data of the Al-doped SiC reveal that the doped $\mathrm{Al}$ causes for the double peaks of Si. With the increasing number of the adsorbed $\mathrm{CO}$ $(1 / 20,1 / 10,3 / 20,1 / 5)$, the adsorption energy of adsorption system decrease linearly which means that the adsorption stability of this system is decreasing with the increasing number of the adsorbed molecules. The bond length and bond angle will increase with the adsorbed CO molecule, and the C atom of $\mathrm{CO}$ will also transfer its role from acceptor to contributor with the second $\mathrm{CO}$ adsorbed. The repellion of the neighbor the same atoms and the next-neighbor effect cause for the selective growing model of odd and even adsorbed $\mathrm{CO}$ adsorbed, which will be investigated in our future works.

\section{Acknowledgments}

This work was supported by the National Natural Science Foundation (NO. 11404257 and 11405127) and the Science research and development project of Shaanxi Province (NO.2016GY-041 and 2015JM1027 ).

\section{Reference}

[1] Casady J B, Johnson R W. Status of silicon carbide (SiC) as a wide-bandgap semiconductor for high-temperature applications: A review[J]. Solid-State Electronics, 1996, 39(10):1409-1422.

[2] Ryu S H, Agarwal A K, Singh R, et al. 1800 V NPN bipolar junction transistors in 4H-SiC[J]. IEEE Electron Device Letters, 2001, 22(3):124-126.

[3] Pollmann J, Peng X, Wieferink J, et al. Adsorption of hydrogen and hydrocarbon molecules on SiC(001)[J]. Surface Science Reports, 2014, 69(s 2-3):55-104.

[4] Kim J, Gila B P, Abernathy C R, et al. Comparison of Pt/GaN and Pt/4H-SiC gas sensors[J]. Solid State Electronics, 2003, 47(9):1487-1490.

[5] Gong A, Yan Z X, Tian Y X, et al. Comparative Study of Physical Adsorption Characteristics of CH_4 on Circular Pore Surface of Porous Silicon(110)[J]. Journal of Materials Science \& Engineering, 2016.

[6] Feng-chun Pan, Zhi-Peng Chen, Xue-ling Wang, et al. Electronic structure and magnetic properties of $(\mathrm{Cu}, \mathrm{N})$-codoped3C-SiC studied by first-principles calculations[J]. Chinese Physics B, 2016, 25(9):458-462.

[7] Jia R X, Zhang Y M, Zhang Y M. First-principle calculation on the defect energy level of carbon vacancy in 4H-SiC[J]. Chinese Physics B, 2010, 19(10):436-440.

[8] Peng X, Krüger P, Pollmann J. Adsorption processes of hydrogen molecules on SiC(001), Si(001) and C(001) surfaces[J]. New Journal of Physics, 2008, 10(12):4036-4042.

[9] Camarda M, Magna A L, Delugas P, et al. First Principles Investigation on the Modifications of the $4 \mathrm{H}-\mathrm{SiC}$ Band Structure Due to the $(4,4)$ and $(3,5)$ Stacking Faults[J]. Applied Physics Express, 2011, 4(2):417-423.

[10] Ohnuma T, Tsuchida H, Jikimoto $\mathrm{T}$, et al. Interface States in SiO2/4H-SiC(0001) Interfaces from First-Principles: Effects of Si-Si Bonds and of Nitrogen Atom Termination[C]// Materials Science Forum. 2005:573-576.

[11] Zhou P L, Shi R Q, He J F, et al. First principle study on B-Al co-doped 3C-SiC[J]. Acta Physica Sinica -Chinese Edition-, 2013, 62(23):233101-233101.

[12] Li Zhi Min Shi Jian Zhang Wei Xiao Hei Li Pei Xian Huang Yun Xia Li Gui Fang Hao Yue School of Technical Physics, University X, An X. First principles calculation of electronic structure for Al-doped 3C-SiC and its microwave dielectric properties[J]. Physics, 2012, 61(23):514-518.

[13] Yan-Kun D, Xin Q, Hai-Bo J, et al. First Principle Study of the Electronic Properties of 3C-SiC 
Doped with Different Amounts of Ni[J]. Chinese Physics Letters, 2012, 29(7):77701-77704(4).

[14] Cheng P. Systematic Theoretical Investigation for Adsorption Behavior of $\mathrm{CO}$ on $\beta$-SiC (001) Surface[J]. Advanced Materials Research, 2013, 634-638(1):3017-3020.

[15] Hooshmand Z, Le D, Rahman T S. CO adsorption on Pd(111) at 0.5ML: A first principles study[J]. Surface Science, 2017, 655:7-11.

[16] Clark S J. First principles methods using CASTEP[J]. Zeitschrift Für Kristallographie, 2005, 220(5/6/2005):567-570.

[17] Lindan P J D. First-Principles Simulation: Ideas, Illustrations and the CASTEP Code[J]. Journal of Physics Condensed Matter, 2002, 14(11):2717-2744.

[18] Kresse G, Furthmüller J. Efficient iterative schemes for ab initio total-energy calculations using a plane-wave basis set.[J]. Physical Review B Condensed Matter, 1996, 54(16):11169-11186.

[19] Javan M B. Adsorption of CO and NO molecules on SiC nanotubes and nanocages: DFT study[J]. Surface Science, 2015, 635:128-142.

[20] Yong-sheng Wang, Zheng-xin Yan, Shao-hua Zhang. The Study of Coordination Adsorption Effect that CO Adsorption on 4H-SiC (001) Surface about Different Placement Angles[C]. AMTIA 2016,11-16.

[21] Peyghan A A, Soltani A, Pahlevani A A, et al. A first-principles study of the adsorption behavior of $\mathrm{CO}$ on Al- and Ga-doped single-walled BN nanotubes[J]. Applied Surface Science, 2013, 270(4):25-32.

[22] Shan B, Zhao Y, Hyun J, et al. Coverage-Dependent CO Adsorption Energy from First-Principles Calculations[J]. Journal of Physical Chemistry C, 2009, 113(15):6088-6092. 